\title{
Study of the Thermal Stability of Ginsengbiomass as a Drying Object
}

\author{
Evgeny S. Koshkalov ${ }^{* 1}$, Irina P. Talipova ${ }^{2}$, Lida A. Feoktistova ${ }^{3}$, Elena P.Barylnicova ${ }^{4}$ \\ KazanFederalUniversity \\ *Corresponding author E-mail: okm206@mail.ru
}

\begin{abstract}
The problem of receiving biologically active medicines of a broad spectrum of activity for the food, pharmaceutical, cosmetic industry is relevant results of a research of heat stability of biomass of a ginseng for the purpose of justification of temperature condition and a way of drying are presented. Influence of duration and temperature of heating on quality indicators of biomass of a ginseng is studied. It is experimentally established that at a temperature of $400 \mathrm{C}$ and durations of heating within 15 hours the maintenance of total glycoside fraction in biomass of a ginseng (SGF) decreases up to 50\%; at a temperature of 1000C and durations of heating within 8 hours of SGF about $30 \%$ decrease. It is revealed that at a temperature of heating up to $400 \mathrm{C}$ with increase in duration of heating increase in speed of process of a thermal inactivation is observed. It is established that the admissible temperature of heating of biomass of a ginseng is $600 \mathrm{C}$. At sublimation drying the maintenance of SGF in dry biomass of a ginseng is 1,5 - 2 times higher, than at convective and vacuum drying. On the basis of results of a research the conclusion is drawn on that sublimation drying is the most preferable way of drying in comparison with vacuum and convective, allowing to receive a dry product with the highest quality indicators.
\end{abstract}

Keywords: ginseng biomass, total glycoside fraction (TGF), heat stability, heating temperature, inactivation, drying.

\section{Introduction}

Dietary supplements of a phytogenesis are widely used as immunoexcitants, an additional source of food and biologically active agents. The food enriched with dietary supplements increase resistance of an organism to various diseases, almost healthy people can use them as prophylactic.

The medicines received from fabrics of herbs of adaptogens $[1,2]$ are of special interest. As one of the most effective remedies of natural origin intended for maintenance of health of the person the ginseng real - Panax ginseng of S.A. Meyer differing in the unique chemical composition is used. The traditional Chinese medicine claims that medicines of a ginseng prolong life and youth. The first mention of a ginseng is dated the XVI BC - in the book by the Aesculapian of traditional Chinese medicine [3]. Originally roots of this plant were used in food, but over time began to read a ginseng as the means restoring forces. This plant was considered a symbol of divine harmony on the earth, and its root reminding a body of the person began to be used as a magic amulet on good luck, prosperity, longevity and fertility.

Medicines on the basis of a ginseng increase operability of an organism, are adaptogens and possess the psychostimulating action, are used for prevention of various diseases of the central nervous system. The ginseng contains various biologically - the active connections causing them medicinal properties. And interesting of them triterpene glycosides are the most unique These are the valuable secondary metabolites having high biological activity $[4,5]$. In domestic pharmacology the ginseng is included in the State Register of Medicines as the powerful toning, all-strengthening, adaptogenic tool increasing immunity, and organism resilience at stressful situations [6].
Ginseng - an endangered species. The wild ginseng can still be met in mountain broad-leaved and coniferous forests of Primorsky Krai (Russia) and in the northeast of China. Plantation cultivation of a ginseng is unprofitable because of complexity of cultivation of a plant in simulated conditions, to slow increase of biomass of a root therefore the reliable source of raw materials capable to provide pharmaceutical, food production, was the industrial technology of receiving biologically active medicines (BAV) created in Russia method of cultures of the isolated fabrics and cages of plants of a ginseng [7].

Advantages of use of biomass of vegetable fabrics in comparison with use of the wild-growing or grown-up raw materials [8]:

- receiving vegetable raw materials regardless of climatic and geographical conditions of growth;

- reduction of time of receiving raw materials: for the cultures of cages the period of cultivation is 25-75 days, for a ginseng - not less than 5 years;

- receiving raw materials, standard on maintenance of BAV; - regulation of growth rate and biosynthesis of BAV.

Stages of technological process of production biologically active drugs as follows: receiving and cultivation of biomass of plant cells and fabrics - receiving dry biomass - receiving and control of biologically active medicines.

Drying is the stage of technological process substantially influencing quality of ready medicine power - and material capacity of production [9].

Now various ways are applied to drying of biological objects: spray-type, drying in the boiling and dense layers, sublimation drying $[10,11]$.

For thermo labile Products to which ginseng biomass belongs by the dehydration method providing high quality of the received dry product with its preservation during it is long time, sublimation drying in a vacuum is. For realization of process and justification 
of parameters of drying it is necessary to consider specific properties of material, in particular, its heat stability.

The heat stability defines the maximum permissible modes of thermal treatment provided that duration of experience corresponds to duration of the used drying method.

The purpose of this work was studying of heat stability of biomass of a ginseng, influence of degree and duration of heating quality indicators of biomass of a ginseng - the total glycoside fraction (TGF), justification of temperature conditions of drying.

\section{Methods}

Object of a research was the ginseng biomass received by method of cultivation of culture of cages on solid agar Murasige nutrient medium - Skuga [12] within 28-30 days of cultivation at a temperature of $25-27^{\circ} \mathrm{C}$.

Stern and Proctor [13] method at which static or isothermal heating of the analyzed sample in the soldered capillaries is carried out were applied to determination of heat stability of biomass of a ginseng.

The hinge plate of biomass of a ginseng weighing $3 \mathrm{~g}$ was placed in the special tight cartridges manufactured of aluminum foil Such amount of biomass was sufficient for carrying out further researches of the analyzed product. Biomass layer thickness in the cartridge was in limits of $1,3-1,5 \mathrm{~mm}$. Cartridges established in special holders and lowered in the thermostat in which the set temperature was maintained. Control of temperature was exercised by the laboratory thermometer with the price of divisions of 0,1 ${ }^{\circ} \mathrm{C}$. After keeping in the thermostat of the cartridge cooled and investigated biomass on the maintenance of SGF.

Temperature of the heating environment in the thermostat was changed from $30{ }^{\circ} \mathrm{C}$ to $100^{\circ} \mathrm{C}$ with an interval of $20^{\circ} \mathrm{C}$. At the set temperature hold time made from 2 to 12 hours. The chosen range of duration of heating corresponded to duration of process of the drying characteristic of the studied methods of dehydration of biological products: convective drying, vacuum dehydration and sublimation. All experiments were made in triple frequency.

\section{Results and discussion}

The thermogram of biomass of a ginseng which analysis visually confirms influence of temperature and a pro-position of heating on the maintenance of the total glycoside fraction (TGF) is provided on the figure 1 . So, if warming up at $40{ }^{\circ} \mathrm{C}$ within $2-10$ hours practically does not reduce biological activity of biomass of a ginseng, then with temperature increase the maintenance of SGF decreases:

- at endurance within 6 hours the maintenance of SGF decreases by $7 \%$;

- at endurance within 15 hours the maintenance of SGF decreases by $50 \%$;

- at temperature increase to 100 the ${ }^{\circ} \mathrm{C}$ in 8 hours the maintenance of SGF decreases to $30 \%$.

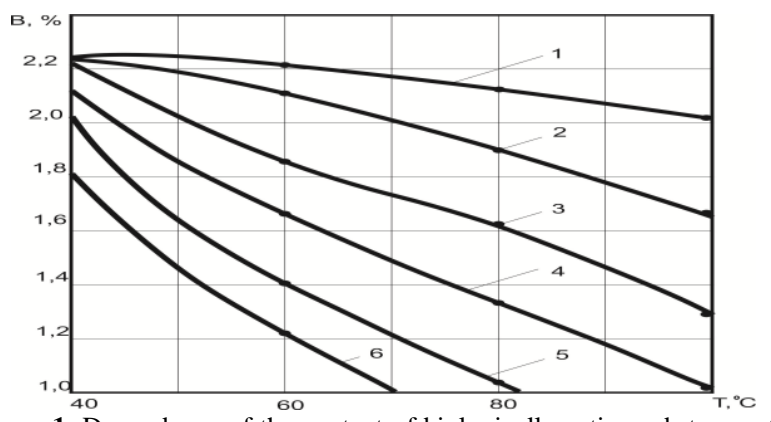

Figure 1: Dependence of the content of biologically active substances (B $\%$ ) on the temperature and duration of heating: $1-\tau=2 \mathrm{~h} ; 2-\tau=4 \mathrm{~h} ; 3-\tau$ $=6 \mathrm{~h} ; 4-\tau=8 \mathrm{~h} ; 5-\tau=10 \mathrm{~h} ; 6-\tau=12 \mathrm{~h}$
For justification of temperature conditions of drying taking into account duration of heating it is necessary to know kinetic parameters of a thermal inactivation of biomass.

The equation of a thermal inactivation can be presented in the form:

$$
\ln \mathrm{B}=-\mathrm{kT}+\ln \mathrm{B}_{0} \ln \mathrm{B}=-\mathrm{kT}+\ln \mathrm{B}_{0},
$$

where In - the maintenance of SGF, \%;

$\mathrm{k}$ - a reaction speed constant, with ${ }^{-1}$

Influence of temperature on the speed of reaction is similar to kinetics of chemical reactions and is described by Arrhenius's equation [14]:

$$
\mathrm{k}=\mathrm{Ae} e^{-\mathrm{Ea} / \mathrm{RT}} \mathrm{k}=\mathrm{Ae}^{-\mathrm{Ea} / \mathrm{RT}},
$$

where And - the preexponential multiplier characterizing a condition of substance and degree of susceptibility to thermal influence with $^{-1}$;

$\mathrm{Ea}$ - energy of an inactivation, $\mathrm{kJ} / \mathrm{mol}$.

Substituting the equation (2) in the equation (1), we will receive the kinetic equation of a thermal inactivation:

$$
\begin{aligned}
& \ln \mathrm{B}=\ln \mathrm{B}_{0}-\mathrm{A} \tau e^{-E a / R T} \\
& \ln \mathrm{B}=\ln \mathrm{B}_{0}-\mathrm{A} \tau e^{-E a / R T}
\end{aligned}
$$

where $\tau$ - heating duration, $\mathrm{h}$.

Kinetic curves of a thermal inactivation of biomass of a ginseng are given in the figure 2 . The comparative analysis of curves demonstrates that the speed of process of a thermal inactivation depending on duration of heating is various. With increase in duration of heating the order of reaction remains, however at $\mathrm{T}=$ $400 \mathrm{C}$ with increase in duration of heating increase in speed of reaction is observed.

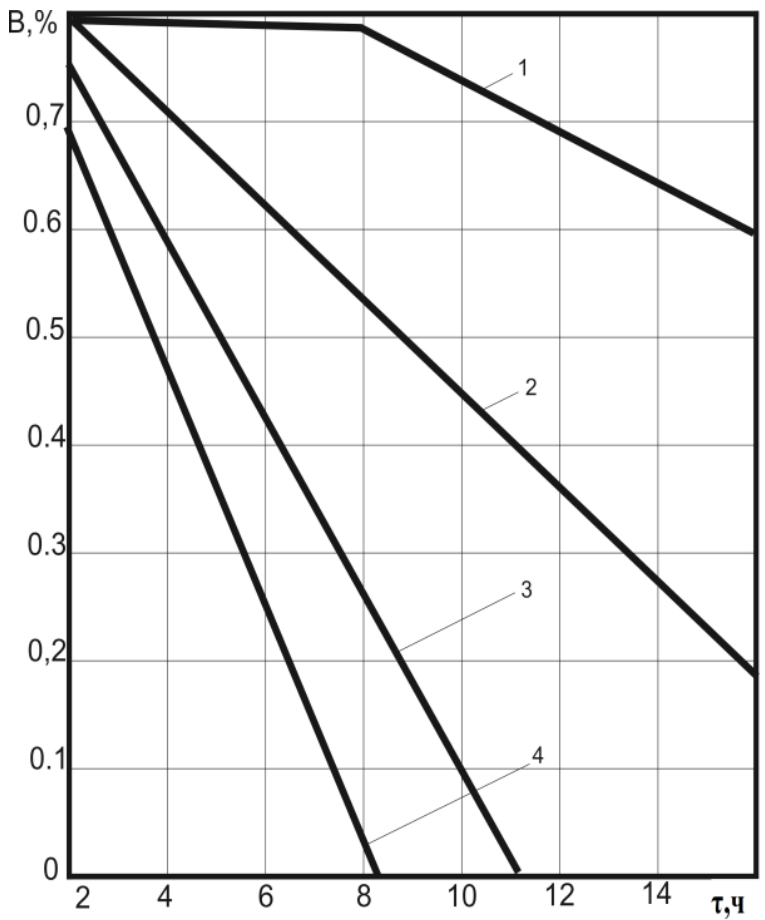

Figure 2: Kinetics of thermal inactivation of ginseng biomass: $1-\mathrm{T}=40^{\circ}$ $\mathrm{C} ; 2-\mathrm{T}=60{ }^{\circ} \mathrm{C} ; 3-\mathrm{T}=80^{\circ} \mathrm{C} ; 4-\mathrm{T}=100^{\circ} \mathrm{C}$

In the figure 3 the dependence of a constant of speed of an inactivation on temperature which linear interpretation is presented in lnk coordinates $=$ to $\mathrm{f}(\mathrm{t})$ is presented. The inflection 
point of a straight line, the corresponding $85^{\circ} \mathrm{C}$, characterizes the most admissible temperature of heating of material.

However more strict justification of the choice of a method and mode of drying taking into account obtaining desirable quality of the final product can is made only in the careful analysis of such heat technical parameters of process of drying as duration and speed of heating and cooling of material, speed of removal of moisture, hygroscopic characteristics.

At the recommendation of any method of drying it is necessary to set the temperature condition of process based on maximum permissible from the point of view of heat stability of material, temperature of the heating environment.

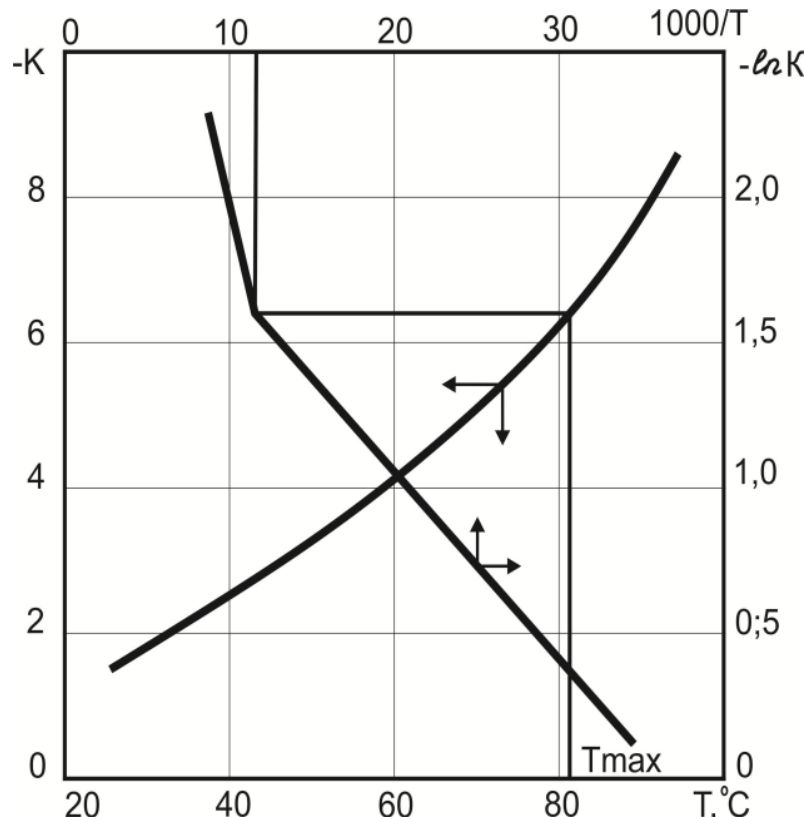

Figure 3: Dependence of the thermal inactivation rate constant on temperature

The most admissible temperature of heating of material at various duration of process is the major technological criterion when developing technology of dehydration of microbiological materials. Increase in duration of heating reduces the most admissible temperature allowing receiving a quality product.In the figure 4 the dependence of the most admissible temperature on heating duration is presented. Apparently from the schedule, in 4 hours the most admissible temperature of heating of material in the course of drying decreases to $600 \mathrm{C}$ and with increase in duration of heating practically does not change.

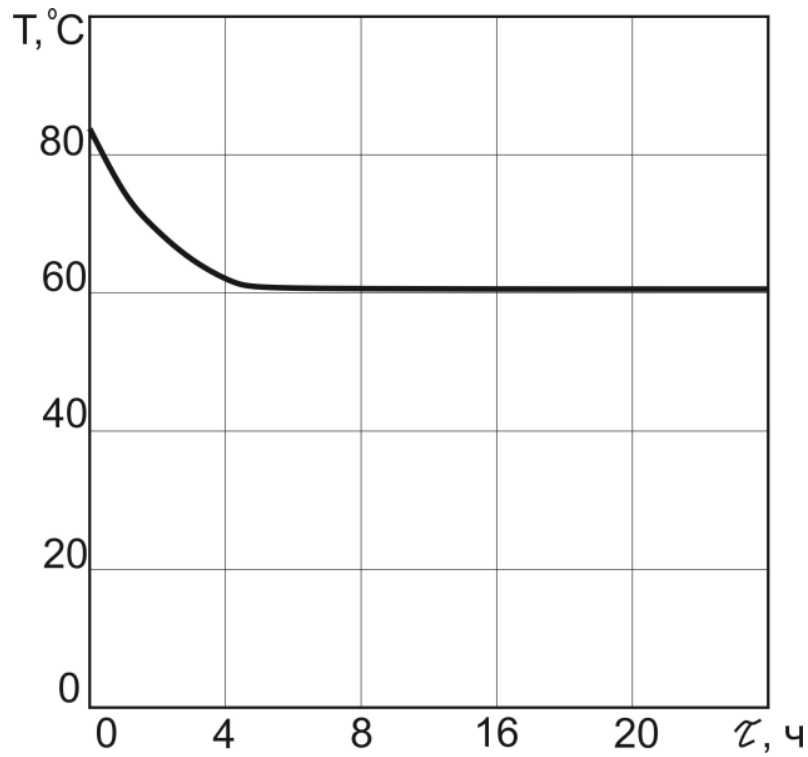

Figure 4: Dependence of the maximum permissible temperature of heating of ginseng biomass on the duration of heating

Therefore, for ginseng biomass drying the most admissible temperature of heating is $+600 \mathrm{C}$.

For justification of a way of drying quality indicators of the biomass of a ginseng which is dried up in various ways were investigated: sublimation, vacuum, convective (atmospheric) at the maximum temperature of heating $+600 \mathrm{C}$ (table 1 ).

Table 1: Effect of drying methods on the quality of dried ginseng biomass

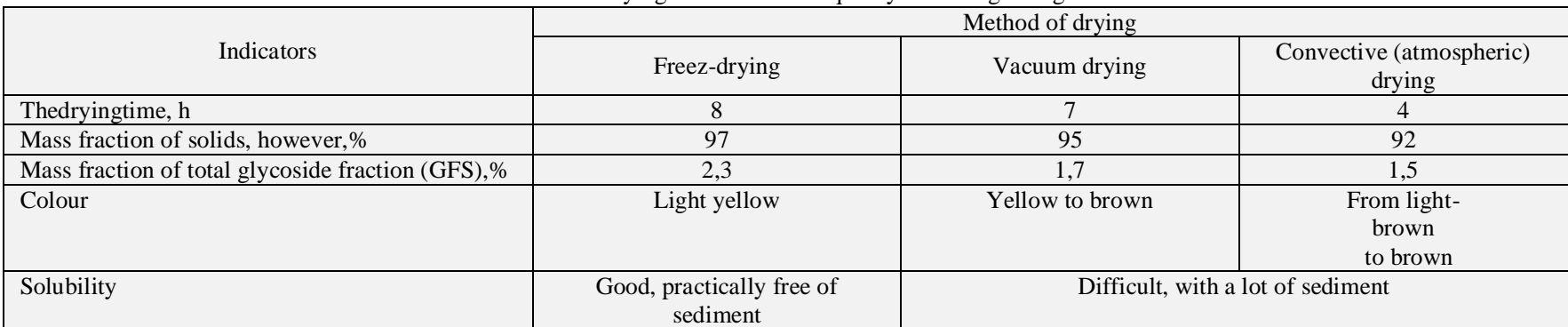

process of a thermal inactivation is observed.

Apparently from the table, at sublimation drying of biomass of a ginseng the maintenance of SGF is 1,5 - 2 times higher, than at convective and vacuum drying and also the solubility of dry biomass improves that increases extractive ability of biomass.

\section{Summary}

1.It is experimentally established that at a temperature of $400 \mathrm{C}$ and durations of heating within 15 hours the maintenance of total glycoside fraction in biomass of a ginseng (SGF) decreases up to $50 \%$; at a temperature of $1000 \mathrm{C}$ and durations of heating within 8 hours of SGF about $30 \%$ decrease.

2.It is experimentally defined that at a temperature of heating up to $400 \mathrm{C}$ with increase in duration of heating increase in speed of
3.It is revealed that the admissible temperature of heating of biomass of a ginseng is $600 \mathrm{C}$.

4.It is established that at sublimation drying the maintenance of SGF in dry biomass of a ginseng is 1,5 - 2 times higher, than at convective and vacuum drying.

\section{Conclusions}

The conducted researches of heat stability of biomass of a ginseng allowed establishing the admissible temperature of heating $+600 \mathrm{C}$. Such temperature condition allows use of different ways of drying, however sublimation drying is the most preferable way in comparison with the vacuum and convective drying allowing receiving a dry product with the highest quality indicators. 


\section{Acknowledgements}

The work is performed according to the Russian Government Program of Competitive Growth of Kazan Federal University.

\section{References}

[1] R.G. Vasilov "A biotechnology role in social and economic development of Russia", the Messenger of biotechnology and physical and chemical biology of Yu.A. Ovchinnikova, T. 4, No. 2 C. 19-22, 2008.

[2] Attele A.S., Wu J. A., Yuan C. - S. "Ginseng pharmacology", Biochem. Pharm. Vol. 58, pp. 1685-1693, 1999.

[3] M.A. Kuznetsov, A.S. Reznikov of "The legend on herbs" - M.: Heigh. school, 1992. - 272 pages.

[4] G.B. Elyakov "A chemical research of suspension culture of cages of a ginseng", Biotechnology, T.5, No. 4, S. 420-426, 1989.

[5] L.I.Slepyan, A.N. Shikov "The success in study and using selected strains of Ginseng - Panaxel and Panasorb", 41st International Congress of Europe Tissue Culture Society, Verona, P. 84, 1994.

[6] Akushskaya, A.S. Standardization of raw materials and medicines of a ginseng. - Akushskaya A. S., Kurkin V. A., Shnytko M.V., Klein L.A. News of the Samara scientific center of the Russian Academy of Sciences. 2012. T. 14. No. 5-3. Page 691-695.

[7] Patent RF No. 2131924 of 16.12.1997. Way of receiving biomass Ginseng/Yakovlev G.P., Galynkin V. A., Slepyan L.I., Zhuravleva D.I.

[8] W.Zhe, J.Zhongjiane.a. "The triterpenoidsaponins of Panax japonicas C.A. Meyer varangustifolius", Actabot.Sinica, Vol.27, No. 6, p.p. 618-624, 1985

[9] J .E .Sunderland "Microwave freeze-drying", Food Process Eng., vol. 4, No. 7, ss. 195-212, 1982.

[10] A.M. Karpov, A.A. Ulumiyev. Drying of products of microbiological synthesis. - M.: Light and food industry, 1987. 224 pages.

[11] T.Kudra, C. Strumillo of "Thermal processing of bio-matherials", Edited by-NewYork: Gordon and breach science publishers, 588 p., 1991.

[12] R.G. Butenko. Biology of cages of the higher plants of invitro and biotechnology on their basis. - M.: FBK-press, 1999.-160 pages.

[13] V.Ya. Munblit, V.L. Talroze, V.I. Trofimov/ Thermoinactivation of microorganisms. - M.: Science, 1985. - 248 pages.

[14] F.Daniels, R. Olberti. Physical chemistry. - M.: World, 1978. - 645 p. 